\title{
Coronal Incision: An Approach to Facial Fractures
}

\author{
Surya Rao Rao Venkata Mahipathy ${ }^{1 *}$, Alagar Raja Durairaj ${ }^{1}$, Narayanamurthy Sundaramurthy ${ }^{1}$ and Shobhan Nandy ${ }^{2}$ \\ ${ }^{1}$ Dept. of Plastic \& Reconstructive Surgery, Saveetha Medical College \& Hospital, Tamilnadu, India \\ ${ }^{2}$ Dept. of Oral \& Maxillofacial Surgery, Saveetha Dental College \& Hospital, Tamilnadu, India
}

\begin{abstract}
Background: The coronal incision with its various modifications provides the most versatile approach to various areas in the craniomaxillofacial region coupled with excellent exposure. The aesthetic advantage of a hidden scar in the hairline, accounts for its continued popularity. In maxillofacial it can be used for fractures in the frontal bone, nasal bone and extensive fractures involving zygomatic arch and complex

Methods: In this clinical and observational study, we operated 5 patients with complex cranio-maxillofacial injuries involving frontal bone, zygomatic arch and zygomatic complex, nasal bone and supra-orbital region. All the five cases were trauma cases between ages of 20 to 60 years with no facial nerve injury per-operatively. These cases for followed for post-operative complications namely sensory and motor nerve deficits, haematoma, wound dehiscence and ptosis

Result: Two patients each had sensory and motor nerve deficits and one patient had minimal wound dehiscence, all settled conservatively

Conclusion: The incision provides excellent access, has reduced complications and produces an acceptable scar. This incision is advised in treating complex cranio-facial trauma, tumors and le-fort esthetic surgeries.
\end{abstract}

\section{Keywords: Coronal, Aesthetic, Facial Fractures, Versatile}

\section{Introduction}

The coronal incision with its various modifications provides the most versatile approach to various areas in the craniomaxillofacial region coupled with excellent exposure. The aesthetic advantage of a hidden scar in the hairline, accounts for its continued popularity. In maxillofacial it can be used for fractures in the frontal bone, nasal bone and extensive fractures involving zygomatic arch and complex. [1]

Severe cranio-maxillofacial injuries especially those involving the mid-face, are difficult to approach, this technique provides optimum exposure of the fracture sites, allowing for accurate anatomic reduction and fixation of the fractured segments and good cosmetic results in the incision site. [2]

This procedure has been used extensively by neurosurgery to gain access to the region of interest. In 1973, Henderson and Jacksondescribed the good access afforded by this flap for Le Fort II osteotomies. It gives excellent exposure to the upper and middle third of facial skeleton. [3]

Various indications for the coronal approach include severe cranio-maxillofacial trauma, craniofacial deformities, craniotomy procedures, osteotomies of upper and middle one third of face, harvesting of bone and fascial grafts when indicated, for improved access to condylar regions, and also for forehead rejuvenation. This procedure has proved to provide adequate exposure even as low as the mandibular condyles without extensive complications. [4]

\section{Materials and Methods}

In this clinical and observational study, we operated 5 patients with complex cranio-maxillofacial injuries involving frontal bone, zygomatic arch and zygomatic complex, nasal bone and supra-orbital region. All the five cases were trauma cases between ages of 20 to 60 years with no facial nerve injury per-operatively.

The positioning of anesthetic tubing for intubation should be in such a manner as to provide optimal access to entire head, face and oral cavity. Out of the 5 cases, three cases went for oral intubation as they did not have any occlusal discrepancies and for the other two we required submental intubation.

A local anesthetic with adrenaline was infiltrated along the planned Lazy S incision line to facilitate dissection and minimize blood loss. The incision was marked 2 to $3 \mathrm{~cm}$ posterior to the hairline extending into the pre-auricular incision. Allis forceps were used to clamp the scalp to achieve hemostasis. The incision was given parallel to hair follicles through the skin, galea, ending superior to the loose areolar plane leaving the periosteum intact. The dissection was carried supra-periosteally and the flap was gradually turned forwards until $5 \mathrm{~cm}$ above the supra-orbital ridges. 
Then the periosteumis incised on the skull and extended over the temporalis fascia. Following that,the dissection is sub-periosteal on the skull and under the temporalis fascia on the temporal region exposing and being superficial to the temporalis muscle. It is important to incise the temporalis fascia just below and along it's attachment to the superficial temporal line. From this point onwards the periosteum and the temporalis fascia is included in the main flap. This step is crucial to prevent facial nerve palsy. This flap is extended down wherethe supra-orbital neuro-vascular bundle was then identified and released from its foramen by removing a small wedge of bone above. This facilitates further retraction of the flap and minimizes paresthesia of forehead. Following complete release of the neuro-vascular bundles, the flap was further dissected infero-medially to expose the entire nasal, ethmoidal and orbital regions. Laterally when the superior border of the zygomatic arch is felt, a subperiosteal incision is made involving the superficial part of the temporalis fascia attachment to the zygomatic arch, exposing the arch and reflecting the entire flap inferiorly. Following that the fractured segments were fixed using appropriate techniques. A drain was then secured which prevented hematoma and the scalp was closed in layers using 3-0 polyglactin and 2-0 nylon. Pressure bandage

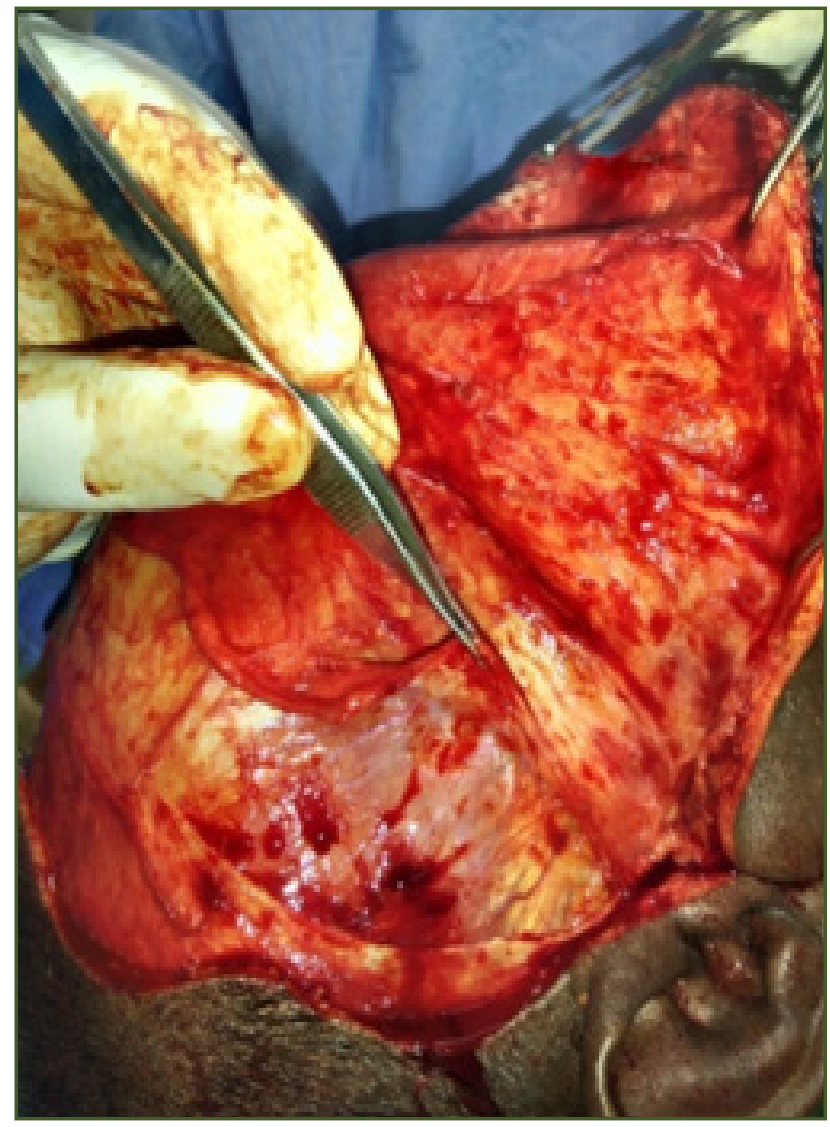

Fig. 1: Temporalis Fascia Elevation. was maintained for 72 hours. Following that the drain was removed and on the fifth post-operative day the patients were clinically examined for the complications. Parameters involved motor nerve deficits, sensory nerve deficits, hematoma, wound dehiscence and ptosis.

\section{Results}

Sensory nerve deficits: 2 out of 5 patients had sensory nerve deficits in the supra orbital and supra trochlear region, but recovered within 2 week post-operatively. They regained complete sensation.

Motor nerve deficits: 1 patient developed frontalis muscle weakness unilaterally, he recovered partially when clinically examined on the $4^{\text {th }}$ follow up week. Another patient developed unilateral frontalis muscle weakness and did not recover even after 4 weeks. So in total 2 patients had this complication.

Hematoma: The drain served well, no patient had this complication.

Wound dehiscence: 1 patient had this complication which required secondary suturing.

Ptosis: No patient was had ptosis.

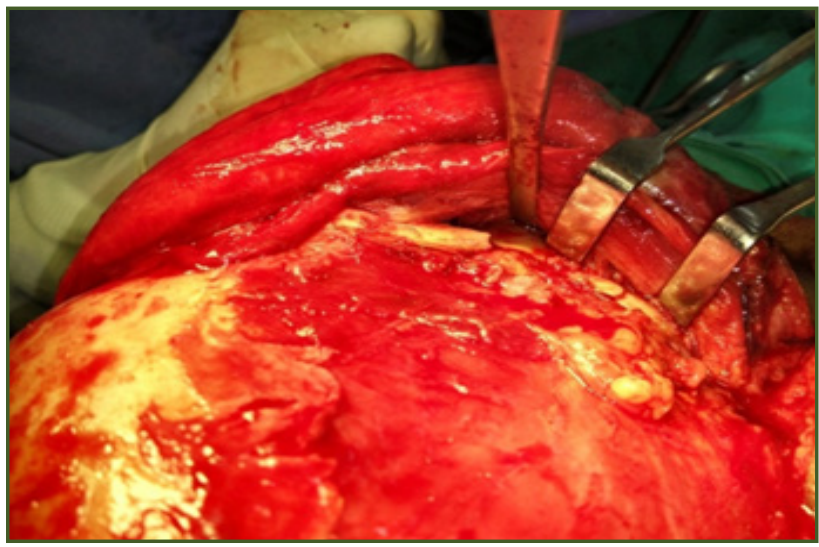

Fig. 2: Exposure of Fractured Zygomatic Arch.

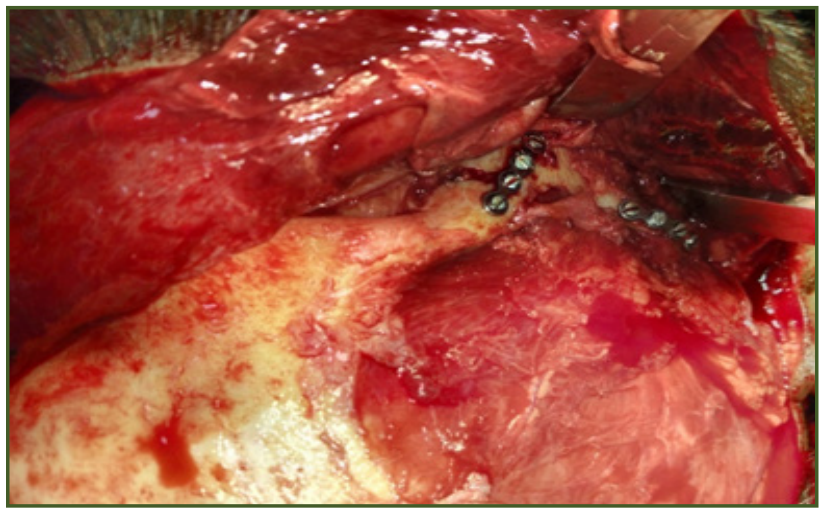

Fig. 3: Fractured Segments Fixed with Miniplates. 




Fig. 4: Fixed Fronto-Zygomatic Region.

\section{Discussion}

The coronal approach traditionally used by the neurosurgeons to gain access to the neurocranium has in the 21 st century has gained popularity in the realm of craniomaxillofacial surgery for exposure of the craniofacial skeleton including the orbit and nasal bones. Since the coronal flap provides access to the frontal, temporal and zygomatic regions, the reconstruction of orbit, zygoma, frontal and nasoorbitoethmoid (NOE) regions is accomplished without the need for any facial incisions. [1]

Although condylar fractures of the mandible may be treated by closed reduction and appropriate physiotherapy, open reduction and internal fixation is indicated in specific circumstances. In a study 25 cases of a previously unreported method of exposure of condylar fractures using an extended bicoronal approach combined with myotomy of the masseter muscle. Acceptable reduction and fixation was achieved in all cases with an early return to function. The incidence of complications was low, with three mild temporary facial palsies which had resolved by the sixth postoperative week and one hematoma beneath the bicoronal scalp flap. A cosmetically acceptable scar was produced in all cases. The excellent surgical exposure and protection of the facial nerve, combined with cosmetically acceptable scars, commend the use of this technique. [4]

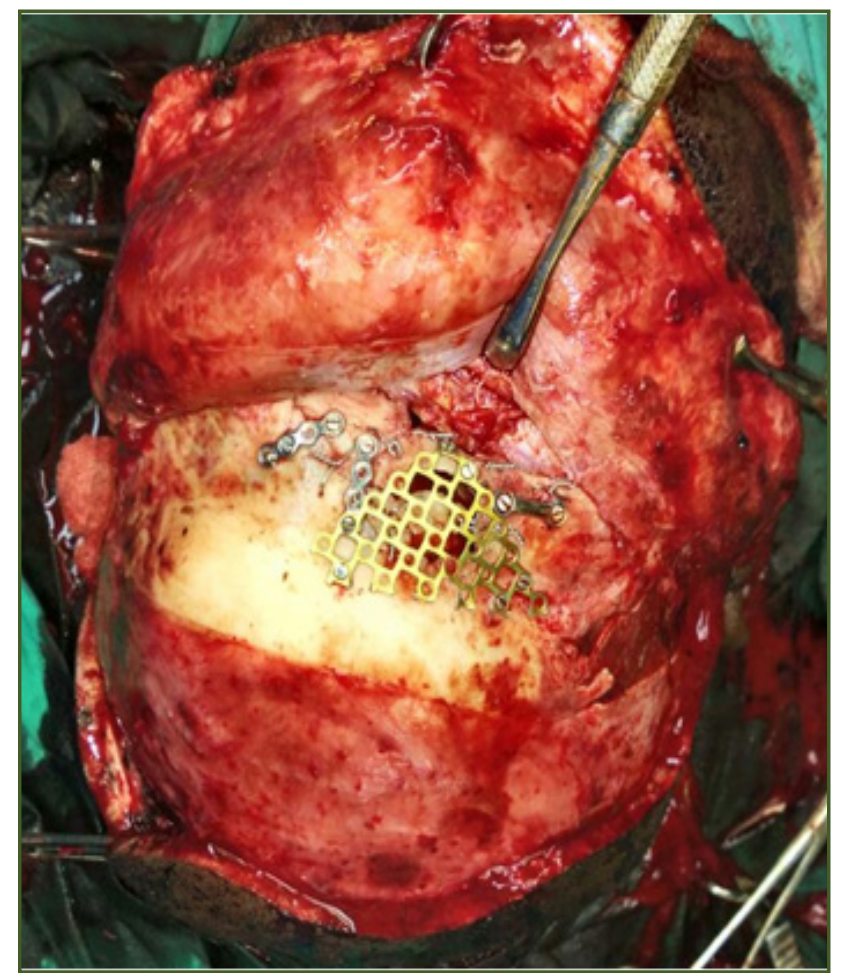

Fig. 5: Flap raised with temporalis fascia and periosteum, Reconstruction of Right Orbital Rim and Frontal bone with Titanium Mesh and Miniplates.

Rejuvenation surgery of the upper one-third of the face can be accomplished by a number of well-known techniques and approaches. This study was a retrospective blinded comparison of pre- and postoperative photographs of patients who underwent forehead lifts. A total of 140 patients having undergone forehead lift procedures and with 12-month postoperative photographic documentation were included in the study. Of these 121 patients had coronal forehead lifts and 19 had endoscopic-assisted forehead lifts. Results revealed that at 1 year follow-up both methods achieved brow elevation without a significant difference in the approach. [5]

Thirty-one cases of comminuted or multiple fractures of the zygomaticomaxillary complex were treated with open reduction and rigid fixation by a coronal approach and analyzed for indications and postoperative complications. Twenty three patients had a hemi coronal approach and eight had a bicoronal approach.

Among the early complications noted were one case of hemorrhage, no infections, and two patients experienced paresthesia/ anesthesia in the supra orbital region, two patients in the temporal/parietal region, six patients experienced facial nerve weakness related to nerve retraction and moderate surgical edema was observed in 
three patients. Late complications included two cases of alopecia/baldness along the incision, one case of persistent paresthesia in the operative area. As far as the esthetics in relation to the incision was concerned, all patients were extremely satisfied. [6]

A retrospective study was conducted on 69 out of 83 patients regarding the indications and complications of scalp incisions for treating zygomatic complex fractures, the other 14 patients were treated by local incisions and approaches.

In the early postoperative period, 5 patients suffered from hemorrhage, 2 had infections, 24 patients reported immediate postoperative anesthesia and paraesthesia affecting the supraorbital region. Six had symptoms and signs of facial nerve injury: difficulty with wrinkling the forehead or to closing the eyes. After a follow-up of 3-5 years, 6 cases suffered from a scar wider than $0.5 \mathrm{~cm}$, paraesthesia in 2 cases (parietal region and temporal region), depression of the temporal fossa in 2 and 1 patient had (persistent) palsy of the temporal branch of the facial nerve. On one hand, coronal incisions offer advantages such as: extensive exposure to ensure exact anatomical reduction. On the other hand, this incision has disadvantages such as obvious scars, long operating time, infections, hemorrhage, paraesthesia in the operative region, palsy of the facial nerve and depression of the temporal fossa. Therefore, the indications for coronal incisions should be strictly applied, and this incision should not be over used. [7]

The coronal flap has recently become a preferred approach for the otolaryngologist-head and neck surgeon requiring access to the craniofacial skeleton and orbit. The variety of cases in which it has proven indispensable include craniofacial reconstruction, facial trauma, and tumor resection. This method of exposure has become particularly useful with increased indications for rigid internal fixation and primary bone grafting in the management of complex facial fractures. Our experience is reviewed in terms of indications for and benefits of the coronal approach, with a detailed description of the technique emphasizing anatomic planes and neurovascular structures. Careful attention to the latter should allow prevention of potential complications. [8]

The coronal incision has been modified so that if it needs to be extended to improve exposure, the extension will be behind the ear and therefore less noticeable. The incision has been used in 25 adults and 30 children with no complications. Its cosmetic appearance is superior to the preauricular coronal incision, and it is preferred especially by young people in whom the scar tends to widen with time. [9]

The coronal scalp incision often leaves a noticeable scar causing the hair to part away from it, especially when wet. Changing the straight-line to a zigzag incision, called the stealth incision, eliminates this obvious deformity. [10]

The use of bicoronal incisions has been sufficiently described in neurosurgery and craniofacial surgery including osteotomies and injuries. This approach provides excellent surgical access for nasal reconstruction with a very low rate of morbidity. A series of 11 patients, together with three case reports, illustrates the advantages and possible complications of this type of incision. [11]

\section{Conclusion}

The incision provides excellent access, has reduced complications and produces an acceptable scar. This incision is advised in treating complex cranio-facial trauma, tumors and le-fort esthetic surgeries. Though complications are rare, but when happens it causes significant morbidity. Thus this should only be indicated only in extensive facial trauma, pathologies and complicated cranio-facial procedures.

\section{References}

1. Rajmohan, Susmitha, David Tauro, Bhupesh Bagulkar, and Anuj Vyas. 2015. "Coronal/Hemicoronal Approach - A Gateway to Craniomaxillofacial Region." Journal of Clinical and Diagnostic Research : JCDR 9 (8): PC01-PC05. doi:10.7860/JCDR/2015/14797.6296.

2. Abubaker, A. O., G. Sotereanos, and G. T. Patterson. 1990. "Use of the Coronal Surgical Incision for Reconstruction of Severe Craniomaxillofacial Injuries." Journal of Oral and Maxillofacial Surgery: Official Journal of the American Association of Oral and Maxillofacial Surgeons 48 (6): 579-86.

3. Shepherd, D. E., R. P. Ward-Booth, and K. F. Moos. 1985. "The Morbidity of Bicoronal Flaps in Maxillofacial Surgery." The British Journal of Oral \& Maxillofacial Surgery 23 (1): 1-8.

4. Dunaway, D. J., and J. A. Trott. 1996. "Open Reduction and Internal Fixation of Condylar Fractures via an Extended Bicoronal Approach with a Masseteric Myotomy." British Journal of Plastic Surgery 49 (2): 79-84.

5. Dayan, S. H., S. W. Perkins, A. J. Vartanian, and I. M. Wiesman. 2001. "The Forehead Lift: Endoscopic versus Coronal Approaches." Aesthetic Plastic Surgery 25 (1): $35-39$.

6. Zhuang, Q.-W., X. P. Zhang, X. Wang, J. Zhang, Z.-P. Li, Y.-M. Si, and J. Meng. 2015. "Coronal Approach to Zygomaticomaxillary Complex Fractures." European Review for Medical and Pharmacological Sciences 19 (5): 703-11. 
7. Zhang, Qing-Bin, Yao-Jun Dong, Zu-Bing Li, and Ji-Hong Zhao. 2006. "Coronal Incision for Treating Zygomatic Complex Fractures." Journal of Cranio-Maxillo-Facial Surgery: Official Publication of the European Association for Cranio-Maxillo-Facial Surgery 34 (3): 182-85. doi:10.1016/j.jcms.2005.09.004.

8. Frodel, J. L., and L. J. Marentette. 1993. "The Coronal Approach. Anatomic and Technical Considerations and Morbidity." Archives of Otolaryngology--Head \& Neck Surgery 119 (2): 201-207; discussion 140.
9. Polley, J. W., and M. Cohen. 1992. "The Retroauricular Coronal Incision." Scandinavian Journal of Plastic and Reconstructive Surgery and Hand Surgery 26 (1): 79-81.

10. Munro, I. R., and J. A. Fearon. 1994. "The Coronal Incision Revisited." Plastic and Reconstructive Surgery 93 (1): 185-87.

11. Atlan, G., P. Jammet, C. F. Schmitt-Bernard, L. Dupoirieux, and F. Souyris. 1994. "Bicoronal Incision for Nasal Bone Grafting." International Journal of Oral and Maxillofacial Surgery 23 (1): 2-5.

*Corresponding author:

Dr. Surya Rao R.V.M., Associate Professor, Dept. of Plastic \& Reconstructive Surgery, Saveetha Medical College \& Hospital, Thandalam, Kanchipuram

Dist. 602105, Tamilnadu, India

Phone: +919444152963

Email: surya_3@hotmail.com 